\title{
Generador autoexcitado: operación en red aislada y sin almacenamiento energético ${ }^{1}$
}

\section{Self-excited generator: off-grid operation without energy storage}

\author{
F. L. Forero, R. Alzate y C. J. Vega \\ Recibido: mayo 05 de 2021 - Aceptado: diciembre 30 de 2021
}

\begin{abstract}
Resumen- El presente artículo aborda el análisis y la simulación de un sistema de generación eléctrica basado en fuentes renovables, operando aislado y sin almacenamiento de energía. Inicialmente, se proponen estrategias de control individual para garantizar regulación en la tensión del bus DC. Para un sistema fotovoltaico, se considera la modificación de un algoritmo de seguimiento del punto de máxima potencia. Para un generador autoexcitado se construye una combinación de conversión mecánica a través de turbina, conversión electromecánica vía generador de inducción, rectificación trifásica y regulación de tensión vía lazo de control PI. A partir de ello, un control droop demuestra un apropiado reparto de potencias entre las diferentes unidades de generación. La topología de sistema propuesta es aplicable como solución de electrificación de bajo costo en zonas rurales de Colombia.
\end{abstract}

Palabras clave - Almacenamiento energético, control droop, generador autoexcitado, sistemas aislados.

Abstract - This article covers the analysis and simulation of an off-grid electrical generation system based on renewable sources without energy storage. First of all, strategies for regulating DC link voltages are proposed for a photovoltaic unit and a self-excited generator. In the first case, a modified maximum power point tracking algorithm has been implemented. For the self-excited generator, a combination of mechanical (turbine), electromechanical (induction machine),

${ }^{1}$ Producto derivado del proyecto de investigación "Control Centralizado para un Sistema de Generación de Energía con Potencial Aplicación en Zonas Rurales del Departamento de Santander", apoyado por la Universidad Industrial de Santander a través del código VIE-UIS 2479.

F. L. Forero, Universidad Industrial de Santander, Bucaramanga, Colombia, email: fabian.forero1@correo.uis.edu.co.

R. Alzate, Universidad Industrial de Santander, Bucaramanga, Colombia, email: ralzatec@uis.edu.co.

C. J. Vega, Universidad del Rosario, Bogotá, Colombia, email: carlosj.vega@urosario.edu.co.

Como citar este artículo: Forero, F. L., Alzate, R. y Vega, C. J. Generador autoexcitado: operación en red aislada y sin almacenamiento energético, Entre Ciencia e Ingeniería, vol. 15, no. 30, pp. 57-62, juliodiciembre de 2021. DOI: https://doi.org/10.31908/19098367.2632.

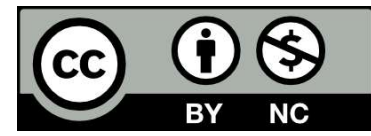

Attribution-NonCommercial 4.0 Intenational (CC By-NC 4.0) electrical (rectifier), and electronic (PI control of power converter) conversions were performed. Hence, after having regulated the input voltage of the power inverter, all the effort relied on a droop controller allowing power-sharing to distribute reasonably the demand among generators of the grid. The proposed system is potentially a low-cost solution for the electrification of rural zones of Colombia.

Keywords - Energy storage, droop control, off-grid system, self-excited generator.

\section{INTRODUCCIÓN}

$\mathrm{E}$ L consumo desmesurado de la sociedad moderna ha provocado impactos negativos en el ambiente, propiciando problemáticas tales como el calentamiento global y el deterioro de recursos naturales, siendo efectos asociados al uso tradicional de recursos fósiles para satisfacer durante décadas la demanda energética global. Adicionalmente, las reservas limitadas de crudo crean oscilaciones en los mercados energéticos haciendo temible la perspectiva económica si se mantiene la dependencia en ese tipo de recurso.

Como alternativa, se plantea la masificación de uso para fuentes energéticas renovables y respetuosas con el ambiente (e.g. solar, hidráulico, eólico o mareomotriz). Colombia posee particular potencial en este tipo de energías debido a su condición natural privilegiada, lo cual permite pensar en un aprovechamiento a gran escala de las mismas. En general, el país cuenta con grandes centrales de generación hidroeléctrica como columna vertebral del sistema interconectado nacional. También es claro que nuestro territorio posee una presencia importante de poblaciones en el área rural, las cuales corresponden mayoritariamente con zonas no interconectadas (ZNI). Algunas soluciones de tipo fotovoltaico (PV) han sido desarrolladas para resolver las necesidades energéticas de pequeñas comunidades bajo estas condiciones. Sin embargo, un sistema PV requiere unidades de almacenamiento para garantizar continuidad del servicio en ausencia de luz, incrementando el costo, tamaño y complejidad de las instalaciones. Asimismo, es posible explotar el alto potencial hidráulico del país a nivel de soluciones de generación a baja escala conocidas como pequeñas centrales hidroeléctricas 
(picohidráulicas, para potencias inferiores a $5 \mathrm{~kW}$ ). Para ello puede aprovecharse el uso de generadores rotativos, que para el caso asíncrono constituyen una solución económica, de fácil mantenimiento y amplia disponibilidad de mercado, especialmente en el tipo jaula de ardilla [1]. Este último se denomina generador autoexcitado (GAE) y requiere que la máquina opere por encima de su velocidad síncrona [2]. De esta manera, es posible acondicionar sistemas de generación combinados que aprovechen recursos fotovoltaicos y picohidráulicos en zonas rurales de Colombia, constituyendo sistemas aislados sin almacenamiento.

De otro lado, operar, controlar y gestionar recursos en sistemas combinados, aislados y sin almacenamiento, genera desafíos a nivel tecnológico y conceptual. Particularmente, existe amplia difusión en la técnica de seguimiento del punto de máxima potencia (MPPT) en sistemas PV [3]. Para sistemas sin almacenamiento la técnica deberá operar, más que en su máxima potencia, en el valor nominal de la demanda a cada instante. Adicionalmente, el reparto de potencias se consigue empleando la técnica denominada control droop [4]. Este método se fundamenta en la inclusión de una impedancia virtual para ajustar las contribuciones de cada generador conectado a la red [5].

Tomando como base las ideas expuestas, el presente artículo propone un sistema de generación aislado sin almacenamiento energético, que combina una unidad PV con un GAE y adicionalmente aborda el control de su operación a nivel de generador individual y de sistema en red mediante una estructura jerárquica basada en la técnica droop.

\section{MARCO CONCEPTUAL}

La máquina de inducción (o máquina asíncrona) es uno de los convertidores electromecánicos de energía más ampliamente difundidos en la actualidad.

A nivel de generación, las actuales necesidades del sistema interconectado por adoptar esquemas basados en fuentes no convencionales de energía, y por consiguiente, con flujos de potencia de característica errática e intermitente, abren la posibilidad para configuraciones asíncronas como el generador autoexcitado, que se destaca como una opción viable en aplicaciones de baja potencia debido a su alta fiabilidad, bajo costo y mantenimiento simple.

Considere un generador conectado a un nodo (o barra) de una red, como se muestra en la Fig. 1.

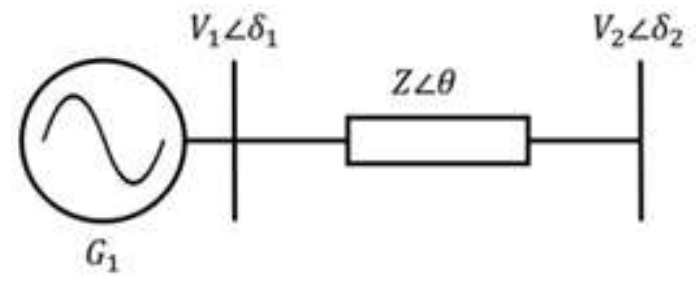

Fig. 1. Generador conectado a una red

La potencia aparente $S$ entregada por el generador a la red, puede calcularse como:

$$
\begin{gathered}
S=V 1 \times I^{*}=\left|V_{1}\right| e^{j \delta_{1}} \times\left(\frac{\left|V_{1}\right| e^{j \delta_{1}}-\left|V_{2}\right| e^{j \delta_{2}}}{|Z| e^{j \theta}}\right)^{*} \\
S=\frac{\left|V_{1}\right|^{2}}{|Z|} e^{j \theta}-\frac{\left|V_{1}\right|\left|V_{2}\right|}{|Z|} e^{j\left(\delta_{1}-\delta_{2}+\theta\right)}
\end{gathered}
$$

A partir de ello, la fórmula de Euler puede ser empleada para generar las siguientes expresiones para las componentes activa (real $P$ ) y reactiva (imaginaria $Q$ ):

$$
\begin{aligned}
& P=\frac{\left|V_{1}\right|^{2}}{|Z|} \cos (\theta)-\frac{\left|V_{1}\right|\left|V_{2}\right|}{|Z|} \cos \left(\delta_{1}-\delta_{2}+\theta\right) \\
& Q=\frac{\left|V_{1}\right|^{2}}{|Z|} \operatorname{sen}(\theta)-\frac{\left|V_{1}\right|\left|V_{2}\right|}{|Z|} \operatorname{sen}\left(\delta_{1}-\delta_{2}+\theta\right)
\end{aligned}
$$

De esta manera, asumiendo una línea de transmisión completamente inductiva $(Z=j X)$ se tendrá $\theta=90^{\circ} \mathrm{y}$ por tanto, ante una variación de ángulo $\phi=\delta_{1}-\delta_{2} \approx 0$, es posible reescribir las expresiones en la forma:

$$
\begin{aligned}
P & =\frac{\left|V_{1}\right|^{2}}{|Z|} \cos \left(90^{\circ}\right)-\frac{\left|V_{1}\right|\left|V_{2}\right|}{|Z|} \cos \left(\phi+90^{\circ}\right) \\
& =\frac{\left|V_{1}\right|\left|V_{2}\right|}{|Z|} \sin (\phi)=\frac{\left|V_{1}\right|\left|V_{2}\right|}{X} \phi ; \\
Q & =\frac{\left|V_{1}\right|^{2}}{|Z|} \operatorname{sen}\left(90^{\circ}\right)-\frac{\left|V_{1}\right|\left|V_{2}\right|}{|Z|} \operatorname{sen}\left(\phi+90^{\circ}\right) \\
& =\frac{\left|V_{1}\right|^{2}}{|Z|}-\frac{\left|V_{1}\right|\left|V_{2}\right|}{|Z|} \cos (\phi)=\frac{\left|V_{1}\right|^{2}-\left|V_{1}\right|\left|V_{2}\right|}{x}
\end{aligned}
$$

tras considerar $\sin (\phi) \approx \phi \mathrm{y} \cos (\phi) \approx 1$, verificando la dependencia explícita de $P$ en la diferencia angular $\phi$ y de $Q$ en términos de las amplitudes de tensión.

Estos resultados constituyen la base para el siguiente conjunto de ecuaciones:

$$
\begin{gathered}
\omega_{i}=\omega_{o}-m_{P_{i}} P_{i} \\
V_{i}=V_{o}-m_{Q_{i}} Q_{i},
\end{gathered}
$$

que definen la técnica de control droop clásico reportado en la literatura, siendo $\mathrm{m}_{\mathrm{P}_{\mathrm{i}}}$ y $\mathrm{m}_{\mathrm{Q}_{\mathrm{i}}}$ las pendientes para las relaciones lineales entre las componentes de potencia y los valores de velocidad $\omega_{i}$ y voltaje $V_{i}$ en el i-ésimo generador.

El objetivo de control en este caso será seleccionar dichas proporciones para ajustar las variaciones de generación en términos de la potencia generada, realizando un reparto equitativo de recursos energéticos a partir de su disponibilidad con respecto a la demanda en un instante dado.

Así entonces, cobra interés la aplicación para la técnica de control droop sobre un generador de inducción autoexcitado en el contexto de un sistema de generación aislado para aplicaciones de baja potencia (microrred).

\section{DESCRIPCIÓN DEL SISTEMA}

La Fig. 2 ilustra la topología de sistema de generación propuesto, presentando una unidad PV en paralelo con un GAE, para alimentar una demanda de $900 \mathrm{~W}$ en una carga puramente resistiva.

A continuación, se aborda la estructura específica de cada 
unidad de generación.

\section{A. Unidad de generación fotovoltaica}

Las etapas constitutivas para el generador PV se muestran en la Fig. 3. Inicialmente, se considera un arreglo de 4 paneles de $250 \mathrm{~W} / 40 \mathrm{VDC}$ en serie, alimentando la entrada de un convertidor de potencia elevador que transforma $160 \mathrm{VDC}$ en su entrada a un valor de 450 VDC en su salida. Posteriormente, esta tensión DC se aplica como entrada (bus DC) a un inversor en puente, que produce en su salida la señal senoidal monofásica de $120 \mathrm{VAC} / 60 \mathrm{~Hz}$, a ser aplicada a la carga. Debido a la presencia de componentes armónicas a la salida del circuito inversor, se realiza un filtrado de paso bajo del tipo LCL [6]. En términos del control de los circuitos convertidores de potencia, para el caso del DC/DC elevador se aplica una técnica MPPT basada en el algoritmo $\mathrm{P} \& \mathrm{O}$ (perturbar y observar) modificado para ajustarse a las condiciones de demanda. Por su parte el inversor de potencia posee un doble lazo de control PI generando la señal de actuación en un modulador SPWM. Para detalles específicos acerca del dimensionamiento de circuitos y el cálculo de parámetros de control para esta unidad de generación, se recomienda al lector interesado consultar [7].

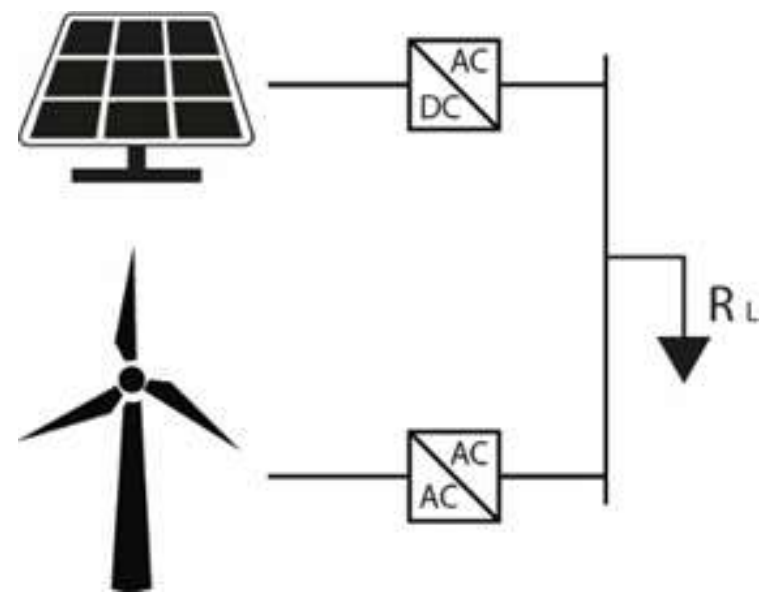

Fig. 2. Topología para sistema de generación propuesto

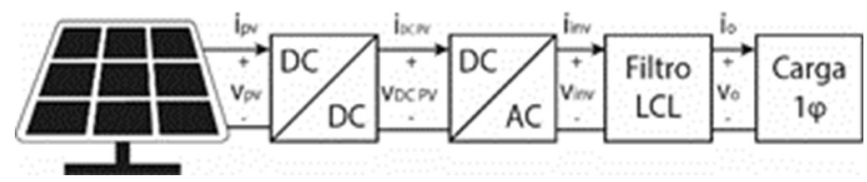

Fig. 3. Unidad de generación PV

\section{B. Unidad de generación rotativa}

El GAE por su parte se constituye de una máquina de inducción que convierte el par de entrada proporcionado por una turbina, en potencia eléctrica trifásica, según se indica en la Fig. 4, donde también se incluye un banco de capacitores en paralelo para proporcionar la potencia reactiva necesaria para magnetizar el campo del estator (autoexcitación) y facilitar así el proceso de generación.

Posteriormente, la tensión trifásica generada es convertida a DC por un rectificador estático basado en diodos y, a partir de allí, la configuración circuital es idéntica al caso del generador PV según se muestra en la Fig. 5.

El dimensionamiento para la unidad GAE se basó en una máquina de inducción real con los siguientes datos de placa: 2 HP, 1700 RPM, 220/440 VAC, 7/3.5 A. Por tanto, a través de un experimento realizado en laboratorio (ver Fig. 6) se pudo determinar la curva de magnetización mostrada en la Fig. 7 relacionando la tensión Vs aplicada en los bornes del estator con la corriente Is circulante a través de sus devanados ante condiciones de vacio. Esta curva es importante, ya que permite validar el modelo matemático a ser empleado en los análisis de simulación numérica del presente artículo.

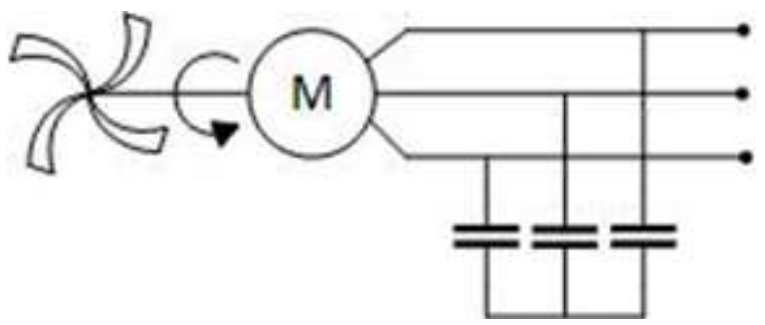

Fig. 4. Generador de inducción autoexcitado

Dicha curva de magnetización, al igual que otros parámetros del modelo equivalente de la máquina de inducción, fueron configurados en MATLAB empleando el bloque para una máquina asíncrona en unidades del sistema inglés, de la caja de herramientas SimPowerSystems de Simulink.

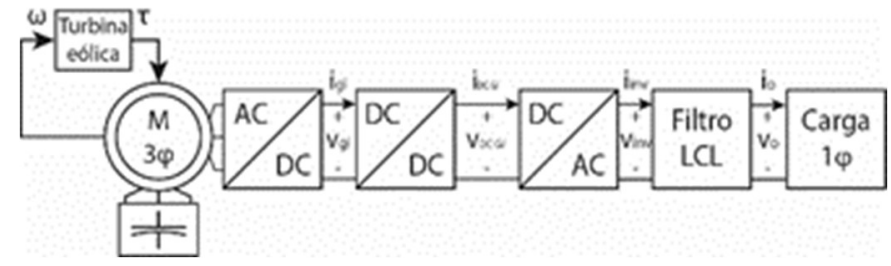

Fig. 5. Unidad GAE

Asimismo, para simular la turbina se utilizó el bloque Wind Turbine disponible en la misma librería, considerando como entradas: 1) la velocidad del viento (valor constante de 12 $\mathrm{m} / \mathrm{s}$ ), 2) la velocidad mecánica del GAE y 3) el ángulo de pitch (asumido como $0^{\circ}$ ). A su vez, el bloque entrega como salida el par aplicado al rotor de la máquina. La configuración del bloque se realizó tomando valores base de potencia de un generador de $1 \mathrm{kVA}$, para operación al $70 \%$ de su capacidad a velocidad nominal del generador. 


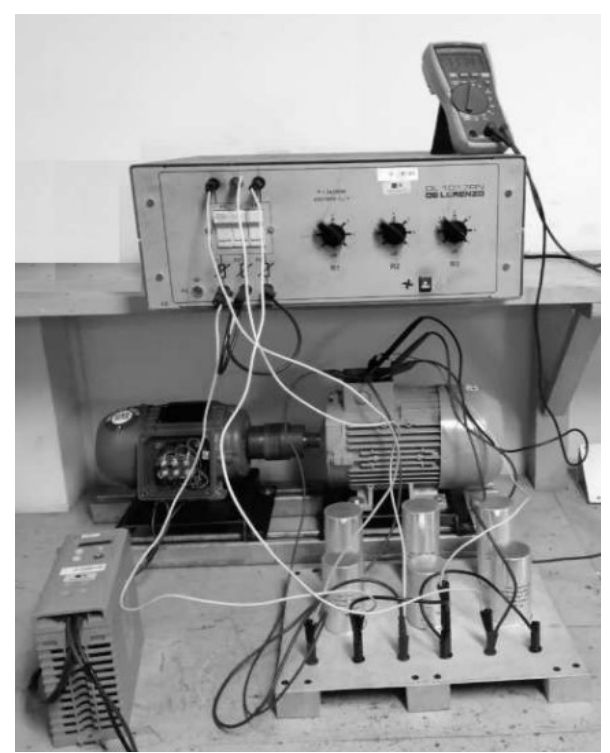

Fig. 6. Experimento de laboratorio para GAE

A partir de lo anterior, se definen los parámetros incluidos en la Tabla I.

\begin{tabular}{lr}
\multicolumn{2}{c}{ TABLA 1: PARÁMETROS GAE } \\
\hline Parámetro & \multicolumn{1}{c}{ Valor } \\
\hline Capacitancia reactivos GAE & $90 \mu \mathrm{F}$ \\
Velocidad nominal del viento & $12 \mathrm{~m} / \mathrm{s}$ \\
\hline Capacitor de entrada DC/DC & $1200 \mu \mathrm{F}$ \\
Pérdidas capacitor de entrada & $0.1 \Omega$ \\
Inductancia DC/DC & $5 \mathrm{mH}$ \\
Pérdidas inductancia DC/DC & $0.2 \Omega$ \\
Capacitor de salida DC/DC & $1100 \mu \mathrm{F}$ \\
Pérdidas capacitor de salida & $0.1 \Omega$ \\
Resistencia acople en bus DC & $22 \mathrm{k} \Omega$ \\
Frecuencia de conmutación & $14 \mathrm{kHz}$ \\
DC/DC & \\
Tensión nominal en bus DC & $500 \mathrm{~V}$ \\
Ciclo útil nominal PWM & 0.5 \\
\hline Frecuencia de conmutación & $14 \mathrm{kHz}$ \\
inversor & \\
Tensión nominal bus de carga & 120 \\
& $\mathrm{VAC}$ \\
\hline Inductancias filtro LCL & $6 \mathrm{mH}$ \\
Capacitancia filtro LCL & $10 \mu \mathrm{F}$ \\
Resistencia de amortiguamiento & $6 \Omega$ \\
\hline
\end{tabular}

Ahora bien, en términos del control de generación se debe tener en cuenta que por tratarse de un generador que opera en forma aislada el flujo de potencia debe ser regulado en función de la demanda requerida por la carga. A partir de ello, siguiendo la metodología presentada en [8] se realizó la sintonización de un lazo de control PI para regular la tensión en el bus DC del GAE. Por su parte, el control del circuito inversor se aborda según detallado a continuación.

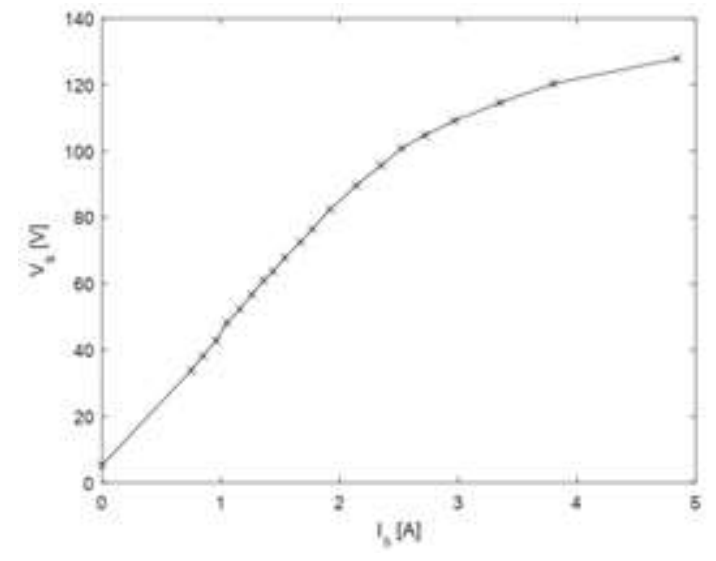

Fig. 7. Curva de magnetización experimental

\section{CONFIGURACIÓN EN RED}

Hasta este punto se ha mostrado la idea general del sistema y las particularidades de generación de cada una de las unidades individuales.

Sin embargo, realizar la conexión paralela de los generadores requiere tomar en cuenta algunos aspectos. En particular, asumiendo una tensión controlada para el bus DC [9], la técnica de control droop se aplica sobre la etapa inversora a partir del esquema general presentado en la Fig. 8.

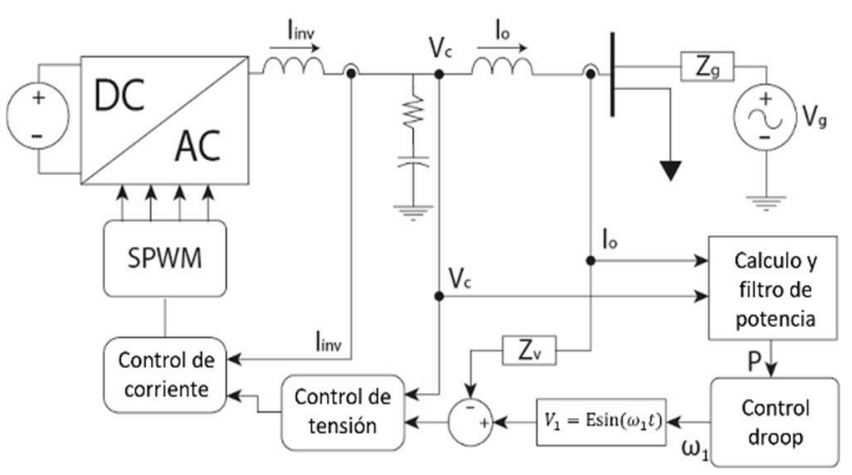

Fig. 8. Esquema droop para inversor monofásico

Fundamentalmente, a partir de (6) la técnica droop (para una carga puramente resistiva como la considerada) parte de la relación lineal entre la diferencia de frecuencias:

$$
\Delta \omega=\omega_{0}-\omega_{1}
$$

y la potencia activa $P$ entregada por un generador a una carga, establecida mediante la expresión:

$$
\omega_{1}=\omega_{o}-m P,
$$

siendo $m$ una pendiente constante, $\omega_{0}$ la frecuencia de la señal senoidal a la salida del inversor y $\omega_{1}$ la frecuencia de la señal en el bus de carga. 


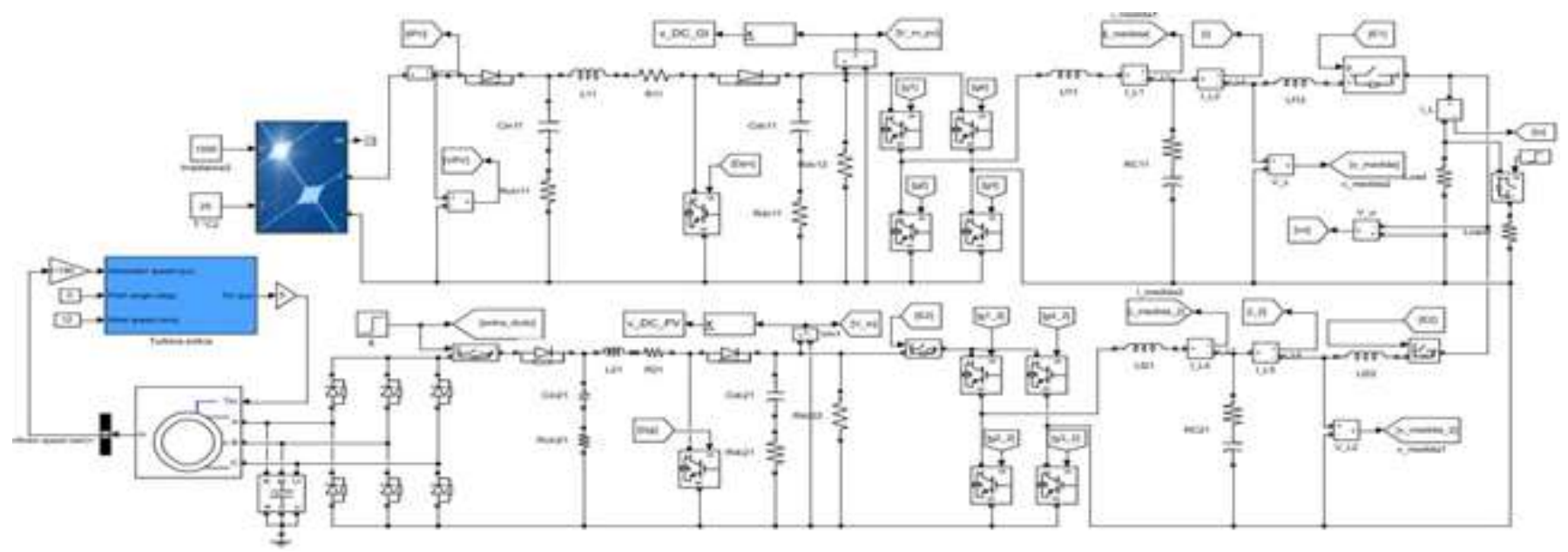

Fig. 9. Implementación circuital para sistema de generación bajo esquema droop

De esta manera, al tener la combinación de dos generadores en red deberán seleccionarse dos pendientes (una para la unidad PV y otra para el GAE) que permitan implementar la proporción de potencias deseada respecto a la demanda de la carga.

La relación lineal considerada es válida ante un acople puramente inductivo entre el generador y el bus de carga. Por tanto, para asegurar esta condición se introduce una impedancia virtual $Z_{V}$.

La implementación circuital definitiva para el sistema de generación bajo el esquema droop se incluye en la Fig. 9. Por su parte, los cálculos detallados y el análisis de la técnica droop pueden encontrarse en [10].

\section{Resultados}

La verificación de operación del sistema se realizó a través de experimentos de simulación desarrollados en MATLAB.

Para ello se construyó un escenario de análisis constituido de las siguientes etapas: 1) los condensadores del bus DC son cargados según se muestra en las Figs. 10 y 11;2) en $\mathrm{t}=1$ [s] se conecta una carga al generador PV que demanda $600 \mathrm{~W} ; 3$ ) en $\mathrm{t}=2.5[\mathrm{~s}]$ se comparte dicha carga con el GAE; 4) en $\mathrm{t}=$ 3.5 [s] la demanda aumenta a $900 \mathrm{~W}$.

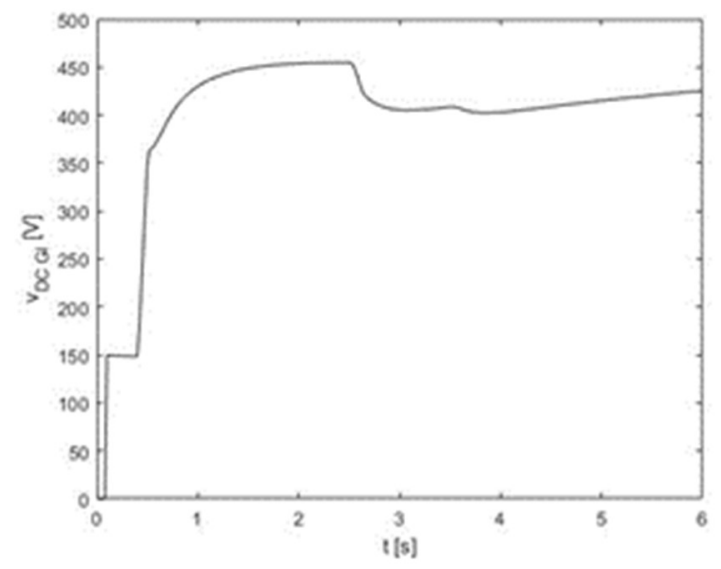

Fig. 10. Tensión bus DC en GAE.
A partir de dichos resultados, se observa la manera en la cual la regulación de la tensión del bus DC es consistente con los valores nominales a la entrada de cada circuito inversor, lo cual facilita obtener una forma de onda deseada en la carga.

Asimismo, de la Fig. 12 se verifica la proporción deseada del reparto de potencias (2/3 GAE $+1 / 3 \mathrm{PV})$ independientemente de los cambios evidenciados en el valor de la demanda, confirmando la efectividad del esquema droop implementado.

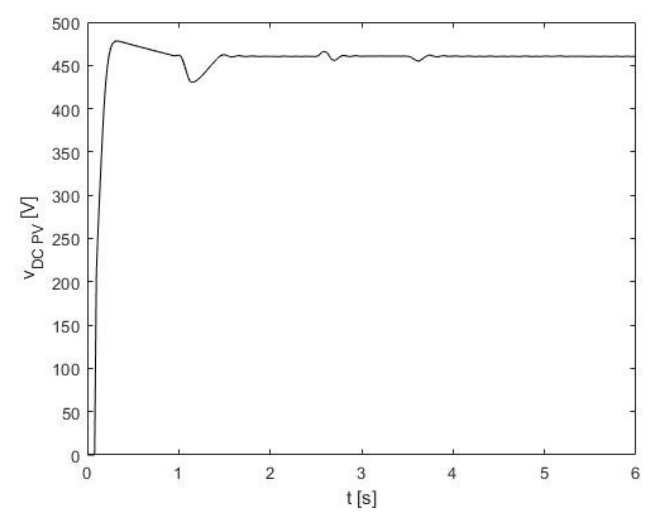

Fig. 11. Tensión bus DC en PV.

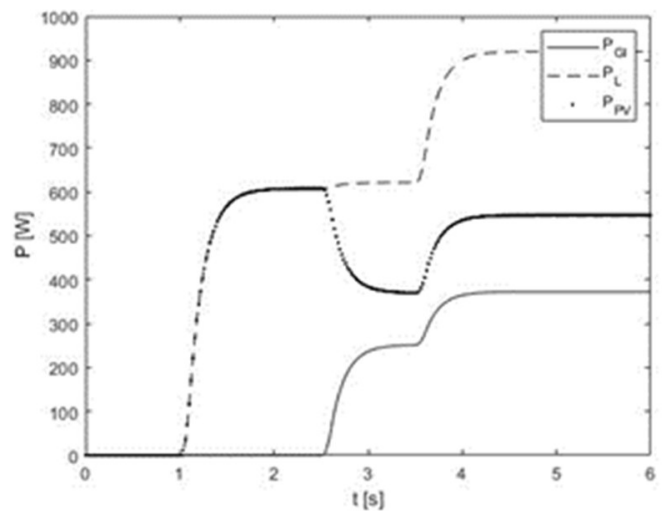

Fig. 12. Reparto de potencias. 


\section{CONCLUSIONES}

El presente artículo propone la combinación en paralelo de dos generadores renovables como caso de estudio para un sistema eléctrico aislado sin almacenamiento de energía. A nivel individual, los generadores presentan estrategias distintas para regular la tensión del bus DC. En el caso fotovoltaico (PV) se realiza un algoritmo modificado de MPPT, mientras para el caso del generador autoexcitado (GAE) se construye una combinación de conversión mecánica a través de turbina, conversión electromecánica vía generador de inducción, rectificación trifásica y regulación de tensión vía lazo de control PI. A partir de dicha condición controlada a la entrada del circuito inversor de potencia, la técnica de control droop demuestra ser un elemento importante para garantizar un apropiado reparto de potencias por parte de las diferentes unidades de generación conectadas a la red, para satisfacer la demanda del sistema, manteniéndose invariante incluso ante diferentes valores de carga. Lo anterior, ratifica la potencialidad de la topología como solución de electrificación de bajo costo en zonas rurales de Colombia, toda vez que representa una solución tecnológicamente viable a partir del desarrollo circuital propuesto para la técnica de reparto de potencias analizada.

\section{AGRADECIMIENTOS}

Los autores agradecen el apoyo de la Universidad Industrial de Santander por financiar las actividades relacionadas con el presente artículo, en el marco del proyecto de investigación VIE-UIS 2479. Adicionalmente, se resalta la contribución de Jaime Andrés Machuca Mayorga en el ajuste de parámetros para el generador autoexcitado, como parte de su trabajo de grado.

\section{REFERENCIAS}

[1] Ortíz-Flórez, R. (2011). Pequeñas centrales hidroeléctricas, Ediciones de la U, Bogotá.

[2] Yaakoubi, A. E., Asselman, A., Djebli, A. y Aroudam, E. H. (2016). A MPPT Strategy Based on Fuzzy Control of a Wind Energy Conversion System, In procedia Technology, Vol. 22, Pages 697-704.

[3] Huang, C., Li, F., y Jin, Z. (2015). Maximum Power Point Tracking Strategy for Large-Scale Wind Generation Systems Considering Wind Turbine Dynamics, IEEE Transactions on Industrial Electronics, Vol. 62, Pages 2530-2539.

[4] Chandorkar, M. C., Divan, D. M. y Adapa, R. (1993). Control of parallel connected inverters in standalone AC supply systems, IEEE Transactions on Industry Applications, Vol. 29, No 1, Pages. 136-143.

[5] Meng, X., Liu., J. y Liu, Z. (2019). A Generalized Droop Control for GridSupporting Inverter Based on Comparison Between Traditional Droop Control and Virtual Synchronous Generator Control, IEEE Transactions on Power Electronics, Vol 34, Pages 5416-5438.

[6] Cossoli, P., Cáceres, M., Vera, L., Firman, A. y Busso, A. (2018). Proportional-Resonant Controller and LCL Filter Design for Single-Phase Grid-Connected PV Micro-Inverters, Pages 1-5.

[7] Forero-Ordóñez, F., Mantilla-Villalobos, M. A. y Alzate, R. (2020). An improved method for power tracking on isolated PV systems without energy storage, IEEE International autumn meeting on power, electronics and computing (ROPEC) 2020. Ixtapa - México. November 2020.
[8] Amaya-Pedraza, A. F., Forero-Ordóñez, F. y Alzate-Castaño, R. (2020). Aplicación de la técnica VRFT como estrategia de control basado en datos, Revista Respuestas. To appear.

[9] He, F., Zhao, Z., Yaun, L., y Lu, S. (2011). A DC-link voltage control scheme for single-phase grid-connected PV inverters, 2011 IEEE Energy Conversion Congress and Exposition, Pages 3941-3945.

[10] Forero, F., Alzate, R., Mantilla, M. y Neves, R. (2020). Off-grid renewable generation control without energy storage, XXIII Congresso Brasileiro de Automática (CBA 2020). Porto Alegre - Brazil. November 2020.

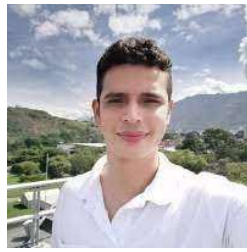

Fabián Leonardo Forero Ordóñez. Ingeniero Electricista (2018) y Magister en Ingeniería Eléctrica (2021) de la Universidad Industrial de Santander en Bucaramanga - Colombia. Posee amplia experiencia en el modelado matemático y el análisis de estabilidad de los sistemas eléctricos de potencia, principalmente aquellos que incorporan recursos renovables de tipo solar fotovoltaico y fluídico (i.e. eólico o hidráulico) a través de microturbinas. ORCID: https://orcid.org/0000-0003-3210-822X.

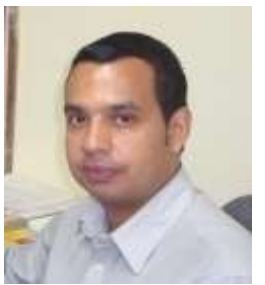

Ricardo Alzate Castaño. Ingeniero Electrónico (2003) y Magister en Automatización Industrial (2006) de la Universidad Nacional de Colombia. Posee un doctorado en Automática de la Universidad de Nápoles Federico II en Italia (2009) y realizó una estancia postdoctoral en la Escuela de Ingeniería de Sao Carlos de la Universidad de Sao Paulo en Brasil (2016). Sus intereses de investigación se centran en la aplicación de técnicas avanzadas de control automático para realizar la gestión apropiada de recursos energéticos en sistemas de baja inercia, a partir del desarrollo de dispositivos basados en electrónica de potencia. ORCID: https://orcid.org/0000-0001-7865-5742.

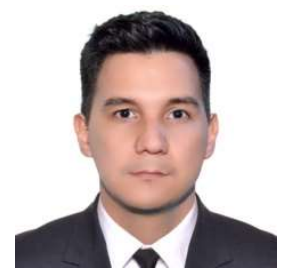

Carlos Jesús Vega Pérez. Ingeniero Electrónico (2014) y Magister en Ingeniería Electrónica (2016) de la Universidad Industrial de Santander en Bucaramanga - Colombia. Es doctor en Control Automático del CINVESTAV - México (2020) y realizó una estancia postdoctoral con la Universidad de Nápoles FEDERICO II en Italia (2021). Sus intereses de investigación se centran en el modelado matemático de sistemas físicos a través de técnicas hamiltonianas, el control óptimo de dinámicas no lineales y la sincronización de sistemas multiagente. ORCID: https://orcid.org/0000-0002-8941-8037. 